Goldschmidt 2021 Abstract

https://doi.org/10.7185/gold2021.3780
7. Lusa et al.(2019), Environ.Res.177,108642.

8. Help et al.(2021), PlantPhysiol.(submitted).

\section{The Ability of Microorganisms to Affect the Behaviour and Mobility of Radionuclides - From Soil to Plants \\ MERJA LUSA}

University of Helsinki, Faculty of Agriculture and Forestry

Presenting Author: merja.lusa@helsinki.fi

Radionuclides present in spent nuclear fuel (SNF) are of major environmental concern and if released, may have damaging consequences for surrounding ecosystems. Microorganisms have fundamental roles in soil biogeochemical cycles and readily respond to the changes occurring in the soil e.g. due to pollution. Microorganisms modify soil chemical environment through various ways including biosorption/-accumulation, bioprecipitation, redox reactions as well as through complexation on excreted ligands (Figure 1). Furthermore, soil microbiota may also induce mobilization of radionuclides e.g. through acidic extracellular metabolites consequently increasing their uptake and transfer to plants.

Considering the effects of microbiota on the behaviour of long-lived radionuclides present in SNF in extreme acidic bog environments, we have observed that the highly diverse microbial communities in the deep anoxic layers tolerate high concentrations of metals and may concurrently contribute to the total retention of radionuclides [1]. The microorganisms present in acidic environments most likely affect radionuclide mobility through several different mechanisms including redox reactions (Se(IV))[2], straight biosorption ( $\mathrm{Ni}(\mathrm{II}), \mathrm{Ag}(\mathrm{I}), \mathrm{Cs}(\mathrm{I}))[1,3,4,5]$ and extracellular enzyme activity (I(-I))[6].

Interactions between soil microorganisms and roots are of major importance to the terrestrial ecosystems. Certain soil bacteria (including Pseudomonas sp. T5-6-I) can increase radionuclide plant-uptake (e.g. transfer of ${ }^{75} \mathrm{Se}$ to Brassica oleracea and Arabidopsis thaliana), and to induce changes in root morphology as well as in protein expression [7]. While generally $\mathrm{Se}(\mathrm{IV})$ reducing $(\mathrm{Se}(\mathrm{IV}) \rightarrow \mathrm{Se}(0))$, Pseudomonas sp. T5-6-I has been shown to increase the transfer of Se(IV) to plants by a hitherto unknown mechanism. However, Se(IV) taken up by the plant seems to end up in organic C-Se-H and C-Se-C bonds regardless of the presence of bacteria [7]. Although interactions between roots and microorganisms are very important, they are often ignored due to the difficulty to image the rootmicroorganism-system. Several novel imaging technologies to image these interactions have however emerged, including stateof-the-art high-resolution synchrotron methods [8].

Figure 1. Microorganism-metal interaction mechanisms References

1. Lusa\&Bomberg (2021), Soil systems (submitted).

2. Lusa et al.(2017), AIMS Microbiol. 3(4),798 -814.

3. Lusa et al.(2016), AIMS Microbiol. 2(2),120-137.

4. Knuutinen et al.(2019), Front.Microbiol. 10, article 2677.

5. Lusa et al.(2015), J.Environ.Radioct.147,22-32.

6. Lusa et al.(2015), J.Environ.Radioct.143,110-122.

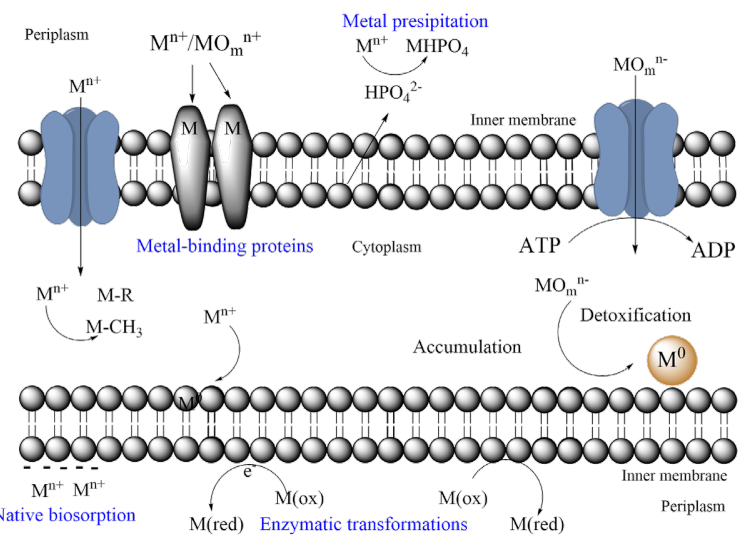

